Voix et Images

\title{
Liminarités et incidences génériques du paysage littéraire québécois du dix-neuvième siècle
}

\section{Réjean Beaudoin et Luc Bonenfant}

Volume 32, numéro 3 (96), printemps 2007

Le dix-neuvième siècle québécois et ses modèles européens

URI : https://id.erudit.org/iderudit/016574ar

DOI : https://doi.org/10.7202/016574ar

Aller au sommaire du numéro

Éditeur(s)

Université du Québec à Montréal

ISSN

0318-9201 (imprimé)

1705-933X (numérique)

Découvrir la revue

Citer cet article

Beaudoin, R. \& Bonenfant, L. (2007). Liminarités et incidences génériques du paysage littéraire québécois du dix-neuvième siècle. Voix et Images, 32(3),

9-15. https://doi.org/10.7202/016574ar d'utilisation que vous pouvez consulter en ligne. 


\title{
L I M I N A R I TÉS ET INCIDENCES GÉNÉRIQUES \\ DU PAYSAGE LITTERAIRE QUÉBÉCOIS \\ DU D IX-NEUVIËME S IĖCLE
}

\author{
$++$ \\ RÉJEAN BEAUDOIN \\ Université de la Colombie-Britannique \\ LUC BONENFANT \\ Université du Québec à Montréal
}

Lors d'un voyage en Europe, Jules-Paul Tardivel notait que "pour un Canadien français, Paris est un monde inconnu; tandis que Londres, c'est tout bonnement la ville de Montréal multipliée par vingt ${ }^{1}{ }$. Cinq ans après son voyage à Paris, Arthur Buies relatait ce qu'il avait vécu en 1867 dans la capitale française, «cette reine des arts et de la pensée dont le nom rayonne sur le monde, éblouissant les imaginations ${ }^{2} »$ :

Paris! C'est un nom qui donne le vertige et j'étais allé me jetter dans le gouffre. Force m'est ici de faire des révélations pathétiques. J'étais seul, sans appui, ignoré, ignorant le sombre et délicieux enfer où s'engloutissent tous les jours tant de vigoureuses espérances. [...] Mon ambition était d'étonner mes contemporains par mon style. [...] Je tombai donc dans cet immense Paris, et dès le premier jour j'eus peur. Il n'est pas de solitude plus déserte qu'une grande ville où l'on ne connaît personne. [...] J'errai, et bientôt je sentis le vide, l'angoisse, le vague saisissement de l'inconnu ${ }^{3}$.

Il est impossible de ne pas remarquer le traumatisme avoué de l'écrivain canadien-français à Paris: la Ville-Lumière porte un «nom [qui] rayonne sur le monde» et "qui donne le vertige»; les périphrases se déploient, amples et nombreuses, autour de ce nom qui s'ouvre aussitôt comme un "gouffre", un "sombre et délicieux enfer». "Le vide, l'angoisse, le vague saisissement de l'inconnu» sont le fait $\mathrm{d}^{\prime}$ « une grande ville où l'on ne connaît personne», mais ce sont aussi les indices d'une aliénation, d'une exclusion plus amère que celle de la communauté des liens du sang ou des rapports sociaux. La nostalgie de l'origine s'exprimait par le sentiment de l'exil sous la plume de François-Xavier Garneau racontant ses propres

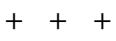

1 Jules-Paul Tardivel, Notes de voyages en France, Italie, Espagne, Irlande, Angleterre, Belgique et Hollande, Montréal, Eusèbe Sénécal, 1890, p. 333-334. 2 Arthur Buies, Chroniques I, édition critique établie par Francis Parmentier, Montréal, Les Presses de l'Université de Montréal, coll. «Bibliothèque du Nouveau Monde», 1986 [1872], p. 335. 3 Ibid., p. 335-336. 
souvenirs de voyage en France: «[C]ette vieille terre [...] dont j'avais tant entendu parler par nos pères, et dont le souvenir se prolongeant de génération en génération, laisse après lui cet intérêt plein de tristesse qui a quelque chose de l'exil $^{4}$.

Une tension frappante se remarque partout dans ces témoignages qui présentent, en effet, un «intérêt plein de tristesse». Il faudra attendre une nouvelle conjoncture et le siècle suivant avant que les écrivains québécois commencent à explorer cet éloignement "qui a quelque chose de l'exil». Et quand Buies raconte cette expérience ( Je tombai donc dans cet immense Paris, et dès le premier jour j'eus peur»), il ouvre un récit encore à venir, récit qui ne pourra s'élaborer que longtemps après lui (chez Marie-Claire Blais, dans Une liaison parisienne ${ }^{5}$, ou chez Michel Tremblay, dans Des nouvelles d'ÉEdouard ${ }^{6}$ ).

Les contemporains d'Arthur Buies étaient douleureusement conscients d'une différentiation nécessaire entre la littérature canadienne-française qu'ils désiraient fonder et la grande littérature française dont le modèle semblait condamner d'avance leurs efforts. On sait qu'Octave Crémazie risqua l'hypothèse suivant laquelle la littérature nationale aurait de meilleures chances de s'imposer si elle s'écrivait en huron plutôt qu' en français ${ }^{7}$. Vers la fin du siècle, Arthur Buies en vint à se demander à qui cette littérature pouvait bien s'adresser chez un peuple "sans classe instruite ${ }^{8}$ ", et Jules Fournier expliqua au critique français Charles ab der Halden ${ }^{9}$, en 1906 et 1907, que la littérature canadienne-française n'existait pas: on avait publié, depuis quelque quatre-vingts ans, des romans historiques et des romans de la colonisation qui se lisaient comme des sermons, des épopées sans héros, des vers réguliers sans poésie et remplis de fierté patriotique, tout cela dans un vide quasi absolu de la critique. Bref, cela ne faisait pas une littérature. En poésie, la consécration de Louis Fréchette à Paris (lauréat de l'un des prix Montyon de l'Académie française en 1880 pour Les fleurs boréales) devait bientôt pâlir devant la révélation d'Émile Nelligan, en 1904, grâce au premier recueil de ses vers réalisé par Louis Dantin: Émile Nelligan et son œuvre ${ }^{10}$. Ainsi, la référence à la France dans la littérature québécoise du dixneuvième siècle semble tout au moins problématique, voire douloureuse.

Dans L'absence du maître, Michel Biron postule que la littérature québécoise se caractérise par cette nécessité de "s'inventer en faisant le deuil du centre ${ }^{11}$ ». Il y montre que l'écrivain liminaire ne souffre pas l'orthodoxie; désinvolte dans ses choix, celui-ci se trouve aussi le plus souvent seul avec lui-même. Les propositions de l'essayiste concernent toutefois au premier chef l'écrivain, dont l'œuvre reflète la

$++$

4 François-Xavier Garneau, Voyage en Angleterre et en France dans les années 1831, 1832 et 1833, édition établie par Paul Wyczynski, Ottawa, Éditions de l'Université d'Ottawa, 1969 [1855], p. 179.5 Marie-Claire Blais, Une liaison parisienne, Montréal, Quinze, 1975. 6 Michel Tremblay, Des nouvelles d'Édouard, Montréal, Leméac, 1984. 7 Lettre d'Octave Crémazie à Henri-Raymond Casgrain, 29 janvier 1867 (Euvres, t. II, texte établi, annoté et présenté par Odette Condemine, Ottawa, Éditions de l'Université d'Ottawa, 1976, p. 90-91). 8 Arthur Buies, I. Réminescences. II. Les jeunes barbares, Québec, Imprimerie de l'Électeur, [1893]. 9 Jules Fournier, «Réplique à M. Ab der Halden », La Revue canadienne, février 1907, p. 128-136. 10 Émile Nelligan, Émile Nelligan et son œuvre, édition préparée par Louis Dantin, Montréal, Beauchemin, 1904. 11 Michel Biron, L'absence du maître. Saint-Denys Garneau, Ferron, Ducharme, Montréal, Les Presses de l'Université de Montréal, coll. «Socius», 2000, p. 308. 
posture à travers les choix esthétiques opérés. À propos de la littérature québécoise du dix-neuvième siècle, nous déplaçons la question afin d'envisager du point de vue esthétique et générique ce que Biron nomme, dans une perspective sociologique, «l'aisance de l'écrivain à passer d'une institution à l'autre, d'une structure à l'autre ${ }^{12}$ ». Autrement dit, comment envisager ce phénomène de liminarité alors que la notion de genre agit comme une mémoire de la littérature ? Contrairement à ce qu'ont voulu croire les romantiques, une œuvre ne naît pas spontanément: elle procède d'emprunts qui sont souvent formels, toujours génériques. Si l'on admet que «la conscience historique, chez des écrivains liminaires [...] ne prend pas la forme d'une séquence linéaire ${ }^{13}$ ", peut-on penser que les genres de la littérature québécoise répondent aussi à un souci de "médiation» plutôt que de domination? Qu'ils ne s'inscrivent pas seulement dans l'ordre complaisant de la reproduction?

Ce rapport générique des deux littératures se complique d'autant plus lorsqu'on examine le paysage de la littérature française en particulier, mais également celui de la littérature européenne en général. Ainsi du roman du dix-neuvième siècle, dont c'est à la fois dire tout et rien que de souligner qu'il est un genre hybride. La caractéristique première de ce genre polymorphe - Charles Baudelaire écrivait «bâtard» - réside dans ce qu'aucune règle ne semble lui appartenir en propre, capable qu'il se montre d'intégrer tous les registres, de se plier à des conventions diverses selon le moment où il est publié (ou l'auteur qui le pratique). Certains l'élèveront au rang de document social: Victor Hugo, dont les romans engagés laissent peu de doute quant au rôle politique dont il dote le genre; surtout Émile Zola, pour qui «le naturalisme est fils de la philosophie positive et matérialiste ${ }^{14}$ ». Georges Rodenbach subsume quant à lui les catégories esthétiques en écrivant ce qui semble alors pourtant impossible: son «roman symboliste», Bruges-la-morte (1892), contrera toutes les attentes en intégrant au sein du régime narratif romanesque les caractéristiques d'un mouvement jusqu'alors exclusivement investi par les poètes. C'est à dessein que nous mettons ici côte à côte Zola et Rodenbach - autrement dit: naturalisme et symbolisme - pour bien marquer l'impossibilité de cerner une fois pour toutes les potentialités génériques du roman du dix-neuvième siècle, pour lequel semble n'exister aucun horizon d'attente spécifique. Seul leur nom générique semble lier des textes aussi différents que NotreDame de Paris (1831) de Hugo, Madame Putiphar (1839) de Petrus Borel, Indiana (1832) de Georges Sand et À rebours (1884) de Joris-Karl Huysmans.

Il serait trop facile de continuer cette liste. Nous apparaît plus important le fait que cette malléabilité du genre n'est pas exceptionnelle dans le paysage littéraire français du dix-neuvième siècle. La crise esthétique produite par le romantisme entraîne une remise en question des normes génériques. Quand il réclame la "déroute du vers alexandrin ${ }^{15}$ ", Stendhal situe en définitive le débat sur le terrain des formes littéraires, auxquelles il demande de représenter «l'état actuel ${ }^{16}$ » de la

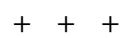

12 Ibid., p. 35.13 Ibid., p. 24.14 Émile Zola, Écrits sur le roman, anthologie établie, présentée et annotée par Henri Mitterand, Paris, Le livre de poche, 2004, p. 301. 15 Stendhal, Racine et Shakespeare. Étude sur le romantisme, édition préparée par Roger Fayolle, Paris, Garnier-Flammarion, coll. «GF», 1970 [1823-1825], p. 76. 16 Ibid., p. 71. 
littérature. Le siècle poétique se fermera d'ailleurs sur l'invention du vers libre. Entre Alphonse de Lamartine et Stéphane Mallarmé, la poésie connaîtra toutes sortes d'avatars, balançant désormais entre le vers et la prose. La notion de genre est donc interrogée, soupçonnée d'une quelconque orthodoxie qui serait contraire à l'esprit du temps, même si les auteurs continuent parfois d'écrire dans des formes et des genres autrement classiques: les "proverbes» d'Alphonse de Musset, le pantoum de Baudelaire, les premiers vers d'Arthur Rimbaud et de Mallarmé en sont des exemples. Ces mêmes Musset, Baudelaire, Rimbaud et Mallarmé produisent pourtant des poèmes en prose, du théâtre de fauteuil ou l'inclassable Coup de dés... Poésie, roman, thêâtre: les trois modes de la littérature française semblent soumis depuis le moment historique du romantisme à des manipulations telles de la part des auteurs et des éditeurs qu'on peut parler, avec Jean-Marie Schaeffer, d'une «thèse romantique de l'a-généricité de la littérature ${ }^{17}$ ». Le siècle de la modernité apparaît comme celui des recommencements génériques, comme le moment fondateur de ce qu'on appellera le «degré zéro» du genre. C'est en lisant les propositions contenues dans la "Préface» de Cromwell ${ }^{18}$, où Hugo défend l'idée selon laquelle le Génie dépasse les règles qui seraient plutôt modelées sur lui, qu'on comprend mieux la formule excessive de Benedetto Croce, pour qui la littérature n'est faite que d'œuvres individuelles puisque "tout véritable chef-d'œuvre a violé la loi d'un genre établi, semant ainsi le désarroi dans l'esprit des critiques, qui se virent dans l'obligation d'élargir ce genre ${ }^{19}$ ».

Cette crise du genre dans tout le dix-neuvième siècle n'équivaut pourtant pas à une éventuelle a-généricité de la littérature, à un refus de la notion de genre. Force est de se rendre compte que les poéticiens parlent de "déroute» (Stendhal) ou d'« élargissement» (Croce), mais jamais d'abolition. Il en va ainsi de Hugo, qui ne se résout pas à tout abattre: «[I]l n'y a ni règles, ni modèles; ou plutôt il n'y a d'autres règles que les lois générales de la nature qui planent sur l'art tout entier, et les lois spéciales qui, pour chaque composition, résultent des conditions d'existence propres à chaque sujet ${ }^{20}$. " L'indétermination générique n'est pas une absence; tout texte appelle nécessairement un horizon générique à partir duquel il devient possible de le lire, car «il n'y a pas de texte sans genre, il y a toujours du genre et des genres mais cette participation n'est jamais une appartenance ${ }^{21} »$.

Dans un tel contexte, comment peut-on envisager le recours aux formes héritées de la littérature française dans l'émergence de la littérature québécoise du dix-neuvième siècle, à la fois en rapport avec cette tradition littéraire et en tenant compte des écarts et des décalages observables en fonction de celle-ci? Comment peut-on mesurer les libertés assumées face aux genres «établis»? Comment est-il possible d'explorer l'érosion et les déplacements des frontières génériques dans les

$$
++
$$

17 Jean-Marie Schaeffer, «Du texte au genre. Notes sur la problématique générique », Gérard Genette et Tzvetan Todorov (dir.), Théorie des genres, Paris, Éditions du Seuil, 1986, p. 202. 18 Victor Hugo, "Préface de Cromwell», Thêâtre complet, t. I, Paris, Éditions Gallimard, coll. "Bibliothèque de la Pléiade», 1963 [1827]. 19 Benedetto Croce, cité par Hans Robert Jauss, «Littérature médiévale et théorie des genres », Gérard Genette et Tzvetan Todorov (dir.), op. cit., p. 41.20 Victor Hugo, op. cit., p. 434.21 Jacques Derrida, «La loi du genre», Glyph, nº 7, 1980, p. 185. 
œuvres aujourd'hui consacrées ou oubliées du dix-neuvième siècle québécois, puisque l'objet de référence apparaît lui-même si flou, et qu'il offre aux littérateurs d'ici un ensemble pratiquement inépuisable de conventions esthétiques et génériques? Autrement dit: que retiennent nos écrivains de l'éventail générique français et européen qui s'offre à eux ? Qu'est-ce que ce choix nous révèle du paysage littéraire québécois du dix-neuvième siècle?

Voilà autant de questions que les articles réunis ici posent, principalement à partir du genre du roman, dont les «libertés» donnent une représentation adéquate de l'indétermination générique du siècle qui nous occupe. La poésie, bien sûr, n'échappe pas à ce rapport européen des genres. Mais l'originalité formelle des versets de Charles Lévesque en font des sortes de hapax au sein de notre poésie, dont les textes principaux laissent le plus souvent poindre une réelle "angoisse de l'influence», pour reprendre l'expression de Harold Bloom. En témoigne l'œuvre de Fréchette qui apparaît comme un cas exemplaire de reconduction servile du modèle de versification hugolien. Le cadre rigide de la versification n'explique qu'en partie ces calques: même le genre poétique en prose reste ici pétri de ce que Jean-Pierre Bertrand appelle, à propos d'Édouard-Zotique Massicotte, une "stéréotypie poétique $^{22}$ ». Chez Silvio, par exemple, la convocation sans cesse répétée de l'intertexte français constitue un appel de l'autorité sur laquelle la création poétique doit $\mathrm{s}^{\prime}$ appuyer pour exister ${ }^{23}$.

Or, l'horizon des genres du récit semble, lui, tout autre. La légende de la Corriveau, telle qu'elle est racontée par José Dubé dans Les anciens Canadiens ${ }^{24}$, renverse dialogiquement les rôles sociaux et l'ordre des valeurs: les détenteurs du savoir enseigné se font clouer le bec par le récit d'un ignorant qui exerce souverainement, tant qu'il détient la parole, le magistère de la sagesse populaire. L'ironisation est tournée contre la parole savante et, notamment, l'histoire, contrairement à ce qui se produira chez des écrivains de la fin du siècle, tels Fréchette et Honoré Beaugrand, qui ne retrouveront les accents de la tradition orale que pour la connoter de naïveté touchante et l'affubler de superstitions insolites. Rien de tel dans les contes de José. Si celui-ci emporte l'adhésion pleine et entière de ses auditeurs, c'est que la culture populaire possédait encore le pouvoir de souder les écarts de classe dans Les anciens Canadiens. Il n'en va certes plus de la sorte dans Pour la patrie ${ }^{25}$, alors que l'affabulation des intrigues politico-mystiques dépasse en invraisemblance toute tradition légendaire. Même si tel n'est pas l'axe de tous les travaux qui se trouvent rassemblés dans ce dossier, il n'en reste pas moins que les études ici proposées traversent, sous des angles chaque fois différents, la prose du dix-neuvième siècle québécois et qu'ils en esquissent indirectement ce qu'on pourrait appeler l'horizon d'attente plutôt fluctuant.

22 Jean-Pierre Bertrand, «Édouard-Zotique Massicotte. La prose de passage», Études françaises, vol. 38, n 3 , 2002, p. 13. Le mérite de Massicotte, selon Bertrand, aura été de servir de «passeur» de la modernité. 23 Voir Luc Bonenfant, «Le poème en prose canadien-français au XIX siècle. L'appel français du genre», Études en littérature canadienne/Studies in Canadian Literature, vol. 30, n 1, 2005, p. 83-99. 24 Philippe Aubert de Gaspé père, Les anciens Canadiens, Montréal, Boréal, coll. «Boréal compact», 2002 [1864]. 25 Jules-Paul Tardivel, Pour la patrie. Roman du XX siècle, édition présentée par John Hare, Montréal, Hurtubise HMH, coll. «Les cahiers du Québec/Textes et documents littéraires», 1976 [1895]. 
Le dossier qu'on va lire s'ouvre par un article de Michel Biron qui étudie la question générique sous l'angle de la formation d'un nouveau lectorat canadien dont la réalité est à construire, problème qui reçoit un éclairage certain par la relecture des Anciens Canadiens de Philippe Aubert de Gaspé père. Ce roman a remporté, comme on le sait, le plus grand succès de librairie de notre dix-neuvième siècle. Or, dès les premières lignes de son livre, le romancier déclare vouloir s'adresser à un public qui ne soit pas strictement restreint aux beaux esprits formés à l'admiration des Belles Lettres. Octave Crémazie et François-Xavier Garneau ne sont pas moins soucieux que lui d'un lectorat local qui ne saurait se définir en fonction d'un milieu littéraire quasi inexistant. Biron étend ainsi son analyse au paysage littéraire ébauché par ces trois écrivains majeurs du dix-neuvième siècle canadien-français, et il montre bien l'importante préoccupation chez eux des différences profondes qui séparent l'Europe littéraire de l'Amérique. Les stratégies d'écriture et les références culturelles, les genres et les formes consacrés doivent par conséquent faire l'objet d'une appropriation et d'une adaptation qui informent les pratiques littéraires du Nouveau Monde, notamment celles des premiers écrivains canadiens-français.

Denis Saint-Jacques s'intéresse à un corpus souvent négligé de nos jours. Son article scrute la prose d'idées, le journalisme naissant et l'élaboration du discours nationaliste, notamment dans l'historiographie puis dans les sciences sociales, depuis le tournant du dix-neuvième siècle jusqu'au début du vingtième, d'Étienne Parent à Léon Gérin. La perspective de l'étude, résolument pluraliste, s'efforce de déboucher sur la synthèse d'influences et de modèles venus de tous les horizons, aussi bien de la France que de l'Angleterre et des États-Unis. Cette clarification offre l'avantage de rappeler l'extrême diversité des différences avec lesquelles nos écrivains ont dû composer leur palette rhétorique et esthétique en métissant leurs codes littéraires.

Micheline Cambron se penche elle aussi sur les formes populaires du journalisme au début du dix-neuvième siècle, plus exactement sur l'interaction de celles-ci avec la prose narrative de nos deux premiers romanciers, Philippe Aubert de Gaspé fils et François-Réal Angers. Le retentissement du crime dans l'actualité journalistique, la recette des faits divers, la contamination réciproque des styles référentiels et fictionnels dans les journaux qui publient alors côte à côte la nouvelle à sensation et le roman-feuilleton - qui se nourrissaient tous deux des mêmes événements -, ces renvois et ces recoupements parfois inextricables entre le reportage et la fiction favorisent la circulation des codes et leur dérive. Après avoir relevé la richesse des références littéraires européennes les plus contemporaines du premier roman canadien-français, Cambron rappelle le caractère bigarré du feuilleton romanesque qui faisait la fortune littéraire de Charles Dickens et de Victor Hugo, dont le succès ne fut pas ignoré des auteurs de L'influence d'un livre et des Révélations du crime. En somme, elle souligne dans cet article le fait peu remarqué que l'esthétique du métissage des codes du journalisme et du roman de cette époque, ainsi que celle qui résulte du mélange des savoirs divers qui s'y rencontrent (alchimie, annales judiciaires, mœurs et légendes populaires), s'impose manifestement à l'attention. Si l'hybridité romanesque vient en partie du choix délibéré de nos auteurs, pareille posture se trouvait déjà autorisée, sinon encouragée par l'horizon d'attente qui était le leur et dont l'inscription se lit clairement dans les œuvres. 
Marie-Andrée Beaudet explore le rôle des ouvrages de piété dans l'écriture de Laure Conan, en interrogeant la transformation de la littérature d'édification spirituelle en roman et en aventure de l'âme féminine dans Angéline de Montbrun. La recherche de Beaudet exhume l'importante bibliothèque de la dévotion doloriste et des pratiques de mortification chrétienne, dont l'archétype se trouve dans L'Imitation de Jésus-Christ. Ce renouveau religieux, qui culmine sous le mandat de monseigneur Ignace Bourget, recourt massivement à l'importation d'une littérature largement diffusée en France. Voici un tout autre modèle dont nous oublions aujourd'hui l'existence, mais dont Laure Conan a choisi d'exploiter les vertus romanesques non seulement dans Angéline de Montbrun, mais dans une grande partie de son œuvre, comme en témoignent sa correspondance et ses écrits intimes.

Jean-Christian Pleau propose une relecture du roman inclassable de JulesPaul Tardivel, Pour la patrie, en mettant au jour les lectures de son auteur, journaliste à La Vérité et polémiste ultramontain qui exerça une grande influence dans la presse canadienne-française de la fin du dix-neuvième siècle. Ardent défenseur des droits de l'Église, Tardivel adhère à l'énorme entreprise de propagande anti-maçonnique qui passionna l'opinion internationale de la fin du siècle. Pleau brosse un tableau très large des lectures de Tardivel romancier, de Léon Taxil à Léon Bloy, sans oublier des auteurs tombés depuis dans une obscurité absolue, comme Paul Deschamps. Si la difficulté de trancher la part du lion de celle des agnelets dans cette constellation $d^{\prime}$ 'influences demeure entière, il n'en reste pas moins que cette recherche nous informe derechef d'une subtile appropriation des modèles français, et des plus inattendus, dans l'écriture de Tardivel.

La diachronie n'est sans doute pas au premier plan de ce dossier, et pourtant, depuis le tout début du siècle jusqu'au tardif roman de Tardivel, y aperçoit-on constamment le déplacement et la modulation des modèles empruntés ou écartés. Encore faut-il faire remarquer la difficulté d'évoquer un horizon d'attente dans un contexte où le lectorat hypothétique correspond, au mieux, à une sorte de pari textuel, ou, au pire, à une réalité manquante. Envisagé sous cet angle, l'horizon générique européen agit comme un horizon herméneutique de la littérature canadienne-française du dixneuvième siècle dont les bancs de nébulosité générique nous apparaissent aujourd'hui comme autant de plages de renouvellement esthétique. 